\title{
Incidence of Malignancy Related Pericardial Effusion in a Tertiary Hospital
}

\author{
NILUFAR FATEMA ${ }^{1}$, WAHIDUR RAHMAN ${ }^{2}$, TASRINA SHAMNAZ SAMDANI ${ }^{2}$, MASADUR RAHMAN ${ }^{2}$, \\ AFSANA RAHMAN ${ }^{2}$, AYESHA SIDDIQUA ${ }^{2}$, AHMED MONJURULAZIZ ${ }^{3}$, MD. ASHRAF UDDIN SULTAN ${ }^{1}$, \\ FARZANASHARMIN², JAHANARAARZU ${ }^{1}$
}

${ }^{1}$ Bangabandhu Sheikh Mujib Medical University, Dhaka, Bangladesh, ${ }^{2}$ Delta Medical College and Hospital, Dhaka, Bangladesh, ${ }^{3}$ Dhaka Dental College Hospital, Dhaka, Bangladesh.

Address of Correspondence: Dr. Nilufar Fatema, Bangabandhu Sheikh Mujib Medical University, Dhaka, Bangladesh E-mail:nilufar.fatema@gmail.com

\section{Introduction:}

Back ground: Malignancy related pericardial effusion (PE) may represent a terminal event in patients with therapeutically unresponsive disease. Pericardial tamponade (PT) is a co morbid condition in many diagnosed malignant cases. Selection of patients with malignancies who are responsive to available treatment may achieve significant improvement in palliation. Early detection of pericardial tamponade and appropriate intervention (Pericardiocentesis) or Subxyphoid pericardiotomy may result long term survival benefit.

Objective: Early detection and management of PE and tamponade in malignant cases.

Methods: Color Doppler (C-D) echocardiography was done in 260 patients with underlying malignancies on chemo or radiotherapy from oncology department of Delta Medical college and hospital from May 2016 to December 2016. These cases were reviewed retrospectively for clinical features, diagnosis and therapy for their malignancy.

Results: $81 \%$ have shortness of breath, sign of pulsus paradoxus and pericardial tamponade were found in $22 \%$

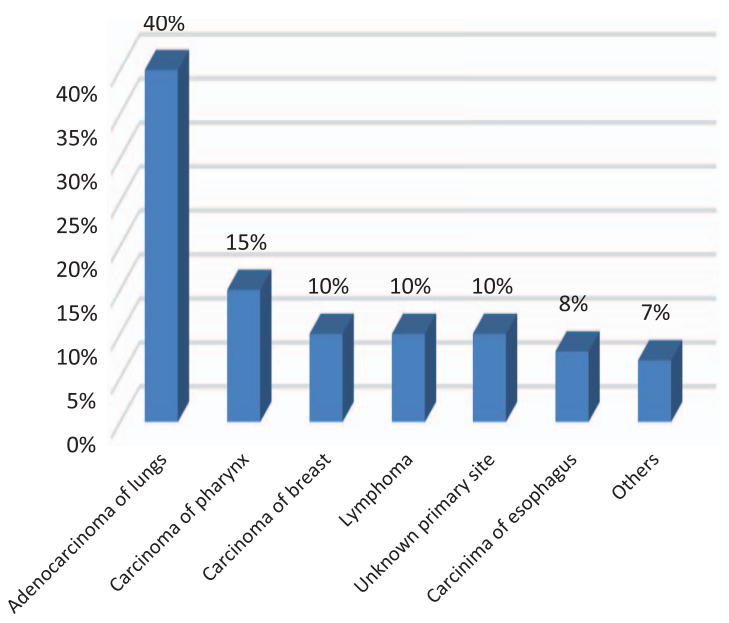

Fig.-1: Causal distribution of pericardial effusion with malignancy

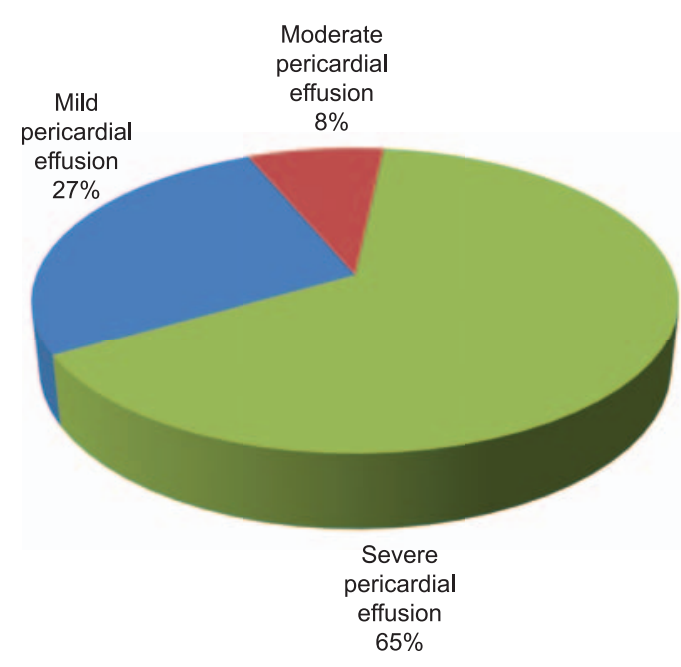

Fig.-2: Extent of pericardial effusion

and 5\% patients respectively. During echocardiography, pericardial effusion was found in 102 patients. $65 \%$ have mild pericardial effusion, whereas $27 \%$ have moderate and $8 \%$ have severe PE. Effusions with malignancy comprising of $40 \%$ adenocarcinomas of lung, $15 \%$ carcinoma $(\mathrm{Ca})$ of pharynx, $10 \%$ Ca breast, $10 \%$ lymphoma, $10 \%$ is Carcinoma of unknown primary, $8 \% \mathrm{Ca}$ esophagus and $7 \%$ others (Renal, Hepatic, ovary \& cheek).

\section{Discussion:}

Malignant pericardial effusion is common feature in patients with disseminated malignancy. The incidence ranges from $5 \%$ to $53 \%$ in autopsy and clinical studies and varies with the histological diagnosis. ${ }^{7}$ Most patients are asymptomatic, but there are chances of developing pericardial tamponade. ${ }^{8}$ Carcinoma of lung, breast, lymphomas, leukemia and melanoma are most common tumors to involve the pericardium. Approximately $75 \%$ of malignant pericardial effusions are clinically diagnosed. ${ }^{9}$ Almost all tumors can potentially involve the pericardium 
and result in an effusion. Malignant pericardial effusion often has an insidious clinical onset that can mimic the picture of disseminated carcinomatosis. Occasionally pericardial involvement may be the first clinical manifestation of an undiagnosed malignant tumor. ${ }^{10}$ Recognizing its presence are particularly important because of the life-threatening potential of cardiac tamponade. Furthermore, in a review of 55 patients with cancer who had pericardial disease, Thurber and co-workers found that pericardial involvement resulted in or contributed significantly to the cause of death in $85 \%$ of patients. ${ }^{11}$ Thus, therapeutic interventions directed at controlling this complication of malignancy can, if successful, result in prolonging survival. The overall survival and quality of life of patients with malignant pericardial effusion is dictated primarily by the histological type and extent of the underlying malignant process. For instance, in three recent large series where patients were treated surgically or with pericardial sclerosis, those with non-small-cell lung cancer had a median survival of approximately three to four months, whereas those with breast cancer had a median survival exceeding nine months. ${ }^{12}$ About $40 \%$ of patients with a symptomatic pericardial effusion and an underlying cancer may have nonmalignant pericardial disease, determining the specific cause is important. In this respect, special attention should be given to those patients who have received prior irradiation to the precordium because the treatment and prognosis of radiation-induced pericarditis are notably different. The differential diagnosis should also include idiopathic pericarditis, infection, hypothyroidism, and autoimmune disorders. ${ }^{13}$ In this report we will not discuss the approach to establishing a specific diagnosis but will focus on the management of patients with a known diagnosis of malignant effusion and outline a treatment plan. The criteria for response vary among series; a direct comparison of the results of each therapeutic modality is not possible. In the few larger series, however, the criteria outlined by Smith and associates were usually used. ${ }^{14}$ These are as follows: a decrease or disappearance of pericardial effusion lasting 30 days or more assessed by radiography and clinical examination; an absence of symptoms of pericardial tamponade for more than 30 days; and no requirement for pericardiocentesis 30 days after the initiation of local or systemic treatment. In more recent reports, echocardiography has been included to augment the accuracy of evaluating response. There are five major methods of treating malignant pericardial effusion: pericardiocentesis, pericardial sclerosis, systemic chemotherapy, radiotherapy and surgical treatment. The overall treatment plan depends on several factors such as the presence of hemodynamic compromise, the general medical condition of a patient, expertise available at a particular institution, and the extent and histological features of tumor. ${ }^{14}$

\section{Conclusions:}

Incidence of malignancy related pericardial effusion was a common feature. The findings of this study also highlight the importance of C-D Echocardiography that could be the investigation of choice for early detection of this condition. Then this group could get appropriate management of malignant pericardial effusion, along with active treatment of the primary cancer. Surgical interventions, such as pericardial window operation, may be warranted in selected patients. So that the long term survival benefit could be achieved.

\section{References:}

1. Cardiac tamponade - unusual clinical manifestation of undiagnosed malignant neoplasm. Perek B, Tomaszewska I, Stefaniak S, Katynska I, Jemielity M. Neoplasma. 2016; 63(4):601-6. DOI: 10.4149/neo_2016_414.

2. Intrapericardial bevacizumab safely and effectively treats malignant pericardial effusion in advanced cancer patients. Chen Dawei, Zhang Yan, Shi Fang, Zhu Hui, Li Minghuan, Chen Kaijun, Kong Li and Yu Jinming Oncotarget. 2016 May 17. doi: 10.18632/oncotarget.9420. DOI: 10.18632/ oncotarget. 9420

3. Prolonged Drainage and Intrapericardial Bleomycin Administration for Cardiac Tamponade Secondary to CancerRelated Pericardial Effusion. Numico G, Cristofano A, Occelli M, Sicuro M, Mozzicafreddo A, Fea E, Colantonio I, Merlano M, Piovano P, Silvestris N: Medicine (Baltimore). 2016 Apr;95(15):e3273. DOI: 10.1097/MD.0000000000003273.

4. Symptomatic Pericardial Effusion After Chemoradiation Therapy in Esophageal Cancer Patients. Junichi Fukada, Naoyuki Shigematsu, Hiroya Takeuchi, Toshio Ohashi, Yoshiro Saikawa,Hiromasa Takaishi,Takashi Hanada, Yutaka Shiraishi, Yuko Kitagawa, Keiichi Fukuda. Presented in part at the 51st Annual Meeting of the American Society for Therapeutic Radiology and Oncology (ASTRO), November 1-5, 2009, Chicago, IL.DOI: http://dx.doi.org/10.1016/ j.ijrobp. 2013.07 .008

5. Neoplastic pericardial disease in lung cancer: Impact on outcomes of different treatment strategies. A multicenter study. Chiara Lestuzzin, Alessandra Bearz, Christos LafarasRenata Gralec,Eugenio Cervesato, Witold Tomkowski,Marzia DeBiasio,Elda Viel, Theodoros Bishiniotis,Dimitrios N. Platogiannis,Angela Buonadonna,Lucia Tartuferi,Rita Piazza, Salvatore Tumolo, Massimiliano Berretta,Francesco Santini,Massimo Imazio Lung Cancer. 2011 Jun;72(3):340 7. DOI: 10.1016/j.lungcan.2010.10.013. Epub 2010 Nov 30.

6. Serous effusions in malignant lymphomas: A review. Dilip K. Das M.B.B.S., M.D., Ph.D., .Sc., F.R.C.Path : Diagn. 
Cytopathol. 2006; 34:335-347. (C) 2006 Wiley-Liss, Inc. DOI: $10.1002 / \mathrm{dc} .20432$

7. The clinical implications of cardiac metastasis from solid tumors-A clinical analysis of 292 cases proved at autopsy. Javier BV,Yount WJ, Hall TC. Neoplasma 1967;14:561-574.

8. Clinical and pathologic features of metastatic neoplasms of the pericardium. Adenle AD, Edwards JE: Chest 1982; 81:166169.

9. Tumors of the heart and pericardium. Scott RW, Garvin CF: Am Heart J 1939; 17:431-436
10. Chen KTK: Extracardiac malignancy presenting with cardiac tamponade. J Surg Oncol 1983; 23: 167-168.

11. Thurber DL,Edwards JE, Achor RWP: Secondary malignant tumors of the pericardium. Circulation 1962; 26:228-241

12. Piehler JM, Pluth JR, Schaff HV, et al: Surgical management of effusive pericardial disease. J Thorac Cardiovasc Surg 1985; 90: 506-516

13. Agner RC, Gallis HA: Pericarditis-Differential diagnostic considerations. Arch Intern Med 1979; 139:407-412

14. Smith FE, Lane M, Hudgins PT: Conservative management of malignant pericardial effusion. Cancer 1974; 33:47-57 